
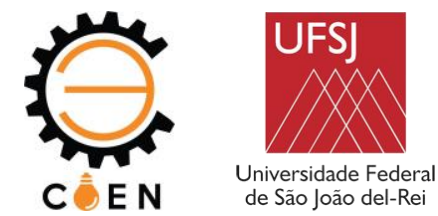

\title{
INFLUÊNCIA DOS ELEMENTOS DE LIGA NA CORROSÃO POR PITE DE AÇOS INOXIDÁVEIS SUPERMARTENSÍTICOS 13Cr EM SOLUÇÃO DE CLORETO A DIFERENTES TEMPERATURAS
}

\footnotetext{
Lucas Nascimento Santana Prachedes ${ }^{(1)}$ (lucasntst@ gmail.com), Caio Vinícius Leão Sabará ${ }^{(1)}$

(caio.leao@oi.com.br), Luan Carrera Santos (1) (luancarrera777@gmail.com), Rhuan Costa Souza (1) (rhuanmecufsj@gmail.com), Lecino Caldeira ${ }^{(2)}$ (lecino.caldeira@ ifsudestemg.edu.br), Gustavo Leitão Vaz

(3) (gustavovaz@ @etrobras.com.br), Jefferson Rodrigues de Oliveira ${ }^{(3)}$

(jefferson.rodrigues@ petrobras.com.br), José Antônio da Cunha Ponciano Gomes ${ }^{(4)}$

(ponciano@metalmat.ufrj.br), Alysson Helton Santos Bueno ${ }^{(1)}$ (alyssonbueno@ufsj.edu.br)

(1) UFSJ - DEMEC - Praça Frei Orlando, 170, Centro, São João del-Rei, MG

(2) IFSudeste - Núcleo de Metalurgia - Rua Bernardo Mascarenhas, 1283, Fábrica, Juiz de Fora, MG

(3) PETROBRAS - CENPES - Av. Horácio Macedo, 950, Cidade Universitária da UFRJ, Rio de Janeiro, RJ

(4) COPPE/UFRJ - METALMAT - Av. Horácio Macedo, 2030, Cidade Universitária da UFRJ, Rio de Janeiro, RJ

RESUMO: Esse trabalho investiga a influência dos elementos de liga na susceptibilidade à corrosão por pite de duas amostras de aços supermartensíticos $13 \mathrm{Cr}$ em solução de $5 \% \mathrm{~m} / \mathrm{v} \mathrm{NaCl}$ em diferentes temperaturas $\left(40,60,80^{\circ} \mathrm{C}\right)$. A caracterização dos aços foi realizada através das técnicas de Microscopia Ótica - MO, Microscopia Eletrônica de Varredura - MEV, Espectroscopia de Energia Dispersiva - EDS e Difratometria de Raios X - DRX. O estudo foi realizado através de ensaios eletroquímicos de polarização potenciodinâmica e espectroscopia de impedância eletroquímica (EIE). A amostra 2 apresentou resistência à corrosão por pite superior a amostra 1 em todas as condições de temperatura. Através das curvas de polarização anódica das amostras, foi observado que o potencial de circuito aberto reduziu com o aumento da temperatura, com exceção da amostra 2 que demonstrou um pequeno aumento na condição de $60^{\circ} \mathrm{C}$ em relação à $40^{\circ} \mathrm{C}$. Em ambas as amostras, o domínio passivo diminui de $40^{\circ} \mathrm{C}$ para $60^{\circ} \mathrm{C}$ e apresenta um aumento na condição de $80^{\circ} \mathrm{C}$ em relação à $60^{\circ} \mathrm{C}$. Adicionalmente, o módulo da impedância decresce com o incremento na temperatura, exceto para a amostra 2 a $80^{\circ} \mathrm{C}$. Apesar de se enquadrarem na mesma classificação, a diferença significativa na fração de elementos de liga presentes, tais como $\mathrm{Cr}, \mathrm{Mo}$, Ti e $\mathrm{Cu}$, contribuiu de forma relevante na resistência à corrosão por pite das amostras, permitindo um melhor desempenho da amostra 2.
}

PALAVRAS-CHAVE: corrosão por pite, aços inoxidáveis supermartensíticos, indústria óleo e gás, cloreto, temperatura, elementos de liga

\section{INTRODUÇÃO}

Nas últimas décadas, o contínuo desenvolvimento da indústria de óleo e gás criou um cenário no qual um transporte seguro da produção é extremamente importante. Visando assegurar a integridade das condições de operação nos oleodutos, as CRA's (Corrosion Resistance Alloys), que exibem uma boa combinação de propriedades mecânicas e resistência a corrosão, são constantemente utilizadas (CONTRERAS et al., 2007; GRUBB e RAKOWSKI, 2016; HILL e PEREZ, 2017; SRIDHAR et al., 2017).

A resistência à corrosão dos aços inoxidáveis se relaciona diretamente com a formação de um filme passivo, o qual age como uma barreira para a difusão das espécies químicas envolvidas nas reações de oxidação. LI et al. (2019) afirma que o filme passivo dos aços inoxidáveis é, em geral, 

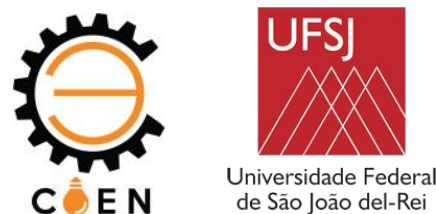

constituído por duas camadas, sendo a camada interna composta por oxido de cromo enquanto a externa é composta por óxidos de ferro ou hidróxidos de acordo com as condições do eletrólito. A capacidade de proteção do filme passivo pode variar de acordo com a adição de elementos de liga e também pelas condições do ambiente de trabalho. Parâmetros como densidade e estabilidade das camadas são profundamente afetados por variações da composição química (ANWAR e ROMIJARSO e MABRURI, 2018; PARDO et al., 2008).

Dentre as CRA's, a aplicação de aços inox martensíticos (AIM) e aços inox supermartensíticos (AISM) é uma opção economicamente viável, uma vez que não é necessário o uso de inibidores ou revestimentos para o controle da corrosão, além de apresentarem um menor custo de produção quando comparados com os aços inoxidáveis duplex (MESQUITA et al., 2014), em especial os AISM que apresentam maior resistência mecânica, dureza, soldabilidade e resistência à corrosão em comparação aos AIM. Essas propriedades podem variar de acordo com o tratamento térmico submetido e composição química (BOJACK et al., 2012).

Os AIM 13Cr incluem aços como AISI 420, com 0,15 a 0,22\%m C e 12 a 14\%m Cr, enquanto os AISM $13 \mathrm{Cr}$ assumem aços com menos que $0,03 \% \mathrm{~m} \mathrm{C}$ e uma adição de molibdênio e níquel (MARCHEBOIS et al., 2006, 2009; MARCHEBOIS e LEYER e ORLANS-JOLIET, 2007). Além disso, o Super $13 \mathrm{Cr}$ não segue regras específicas como os aços $13 \mathrm{Cr}$ convencionais.

TAKABE et al. (2009) afirma que os AISM 13Cr, os quais são categorizados como "13-5-2" de acordo com a ISO 13680 (2010), foram desenvolvidos como materiais de alta resistência a fragilização causada por sulfeto em comparação aos aços inoxidáveis martensíticos $13 \mathrm{Cr}$ convencionais de similar resistência mecânica (AISI420). Além disso, a norma ISO 15156-3 (2015) aponta que qualquer combinação de temperatura e concentração de cloreto que esteja presente no ambiente de produção é aceitável para o uso dos aços 13Cr e S13Cr. No entanto, NICE e MARTIN (2005) observaram que a concentração de $\mathrm{Cl}^{-}$possui uma influência significante na resistência a fragilização causada por sulfeto do Super $13 \mathrm{Cr}$, criando a necessidade de avaliar os limites do seu uso em um ambiente com altas concentrações de $\mathrm{Cl}^{-}$e temperaturas elevadas.

Normas como as ASTM A743/A743M (2014), DIN EN 10088-1 (2014), ISO 13680 (2010), determinam a faixa de composição específica para cada liga, logo é essencial compreender como variações dentro dessas determinadas faixas afetam a resistência à corrosão dos materiais.

Apesar do alto custo e disponibilidade limitada (JUNG et al., 2018), o molibdênio é amplamente utilizado para aprimorar a resistência à corrosão dos aços inoxidáveis em altas concentrações de $\mathrm{Cl}^{-}$. HASHIMOTO et al. (1979) sugere que o Mo enfraquece o processo de corrosão por pite pela eliminação de pontos de superfícies ativas através da formação de óxidos e hidróxidos de Mo nos pontos de difícil formação de filmes passivos. Outros autores ainda concluem que o Mo pode não atuar diretamente na obtenção de filmes passivos, mas contribui pelo enriquecimento de $\mathrm{Cr}$ e formação de óxidos de Cr (HABAZAKI et al., 1992; TAN et al., 1995).

Outro elemento de liga que afeta a resistência à corrosão dos aços inox é o titânio. RODRIGUES et al. (2007) demonstra que o Ti quando utilizado como elemento de estabilização e refino possui um efeito positivo na resistência à corrosão através da formação de um carbonitrido estável, Ti(C,N), após determinado tratamento térmico. Entretanto, adições acima de $0.2 \% \mathrm{~m}$ Ti levam a precipitações de compostos intermetálicos, tais como TiNi, ao invés desse carbonitrido benéfico à resistência à corrosão por pite.

Adicionalmente, o cobre é um elemento de liga que afeta de forma indireta a resistência à corrosão por pite. Segundo YE et al. (2012) o efeito do $\mathrm{Cu}$ relaciona-se com sua função como 

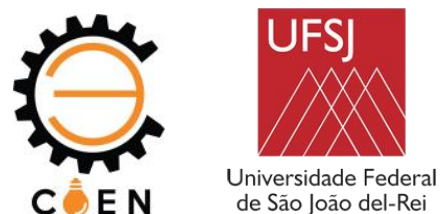

estabilizador e de refino da austenita, promovendo a formação de austenita reversa, microestrutura favorável à resistência à corrosão.

Dessa forma, definir os elementos de liga presentes nos aços, especialmente, $\mathrm{Cr}$, Mo, $\mathrm{Ti}$ e $\mathrm{Cu}$, é crucial no entendimento do comportamento corrosivo desses materiais. Esse trabalho tem como objetivo analisar os diferentes papéis que os elementos chave possuem no processo de corrosão por pite do Super $13 \mathrm{Cr}$ em solução de cloreto e variadas temperaturas.

\section{MATERIAIS E MÉTODOS}

\subsection{Caracterização química e metalográfica}

Os materiais analisados foram os aços inox supermatensíticos (AISM), com 2 amostras de Super 13Cr. A caracterização química dos espécimes (Tabela 2) foi conduzida utilizando espectrometria com emissão ótica e comparadas com base em duas normas, apresentadas na Tabela 1. Para a caracterização metalográfica, os corpos de prova foram observados através de um microscópio ótico, modelo Olympus BX51, com um sistema de aquisição de imagem digital. Adicionalmente, as amostras foram caracterizadas através de um Microscópio Eletrônico de Varredura (MEV), modelo Hitachi TM 3000, além da identificação dos elementos presentes em precipitados observados nas amostras, realizada com a técnica de Espectroscopia de Energia Dispersiva (Energy Dispersive Spectroscopy - EDS). As fases que compõem a microestrutura dos corpos de prova foram analisadas através da técnica de Difratometria de raios X (DRX), com o difratômetro de raios X modelo Shimadzu XRD-6000. Os dados foram capturados na faixa $2 \theta$ de 10 $85^{\circ}$ com uma varredura de $2^{\circ}$ por minuto.

Antes das análises, as amostras foram preparadas utilizando lixas SiC (granulações de 120 até 1500), polidas com Alumina (0,3 um) e então atacadas quimicamente com Vilella (100 ml etanol, $5 \mathrm{ml}$ de $\mathrm{HCl}$ e $1 \mathrm{~g}$ ácido pícrico) por 10 minutos.

Tabela 1. Limites de composição química estabelecidos pelas normas

\begin{tabular}{|c|c|c|c|c|c|c|c|c|c|c|c|}
\hline Amostra & $\begin{array}{c}\text { Identificação } \\
\text { da Norma }\end{array}$ & $\begin{array}{l}\text { C máx } \\
\text { C \%m }\end{array}$ & $\begin{array}{l}\text { Cr máx } \\
\text { Cr\%m }\end{array}$ & $\begin{array}{c}\mathrm{Ni} \\
\text { máx } \\
\mathrm{Ni} \\
\% \mathrm{~m}\end{array}$ & $\begin{array}{l}\text { Mo } \\
\text { máx } \\
\text { Mo } \\
\% \text { m }\end{array}$ & $\begin{array}{c}\mathrm{Si} \\
\text { máx } \\
\mathrm{Si} \\
\% \mathrm{~m}\end{array}$ & $\begin{array}{c}\mathrm{P} \\
\text { máx } \\
\mathrm{P} \\
\% \mathrm{~m}\end{array}$ & $\begin{array}{c}\mathrm{S} \\
\text { máx } \\
\mathrm{s} \% \mathrm{~m}\end{array}$ & $\begin{array}{c}\text { Mn } \\
\text { máx } \\
\text { Mn } \\
\% \text { m }\end{array}$ & $\begin{array}{c}\mathrm{N} \\
\text { máx } \\
\mathrm{N} \\
\% \mathrm{~m}\end{array}$ & $\begin{array}{c}\text { Outros máx } \\
\% \text { m }\end{array}$ \\
\hline $\begin{array}{c}\text { AISM } \\
13 \mathrm{Cr}\end{array}$ & UNS S41426 & 0,03 & $\begin{array}{c}11,5 \text { até } \\
13,5\end{array}$ & $\begin{array}{l}4,5 \\
\text { até } \\
6,5\end{array}$ & $\begin{array}{c}1,5 \\
\text { até } 3\end{array}$ & 0,5 & 0,02 & 0,005 & 0,5 & - & $\begin{array}{c}\text { Ti } 0,01 \text { até } 0,5 \\
\text { V } 0,5\end{array}$ \\
\hline
\end{tabular}

\subsection{Avaliação da resistência à corrosão}

Os testes eletroquímicos foram realizados em uma célula eletroquímica convencional com três eletrodos: um contra eletrodo de platina, um eletrodo de referência de calomelano saturado (ECS) e um eletrodo de trabalho. Os eletrodos de trabalho foram preparados por lixamento em SiC (granulações de 120 até 600), limpos em acetona, secos com ar frio, e expostos por $24 \mathrm{~h}$ ao ar para repassivação. 

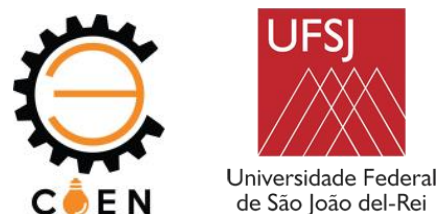

A Polarização Potenciodinâmica e a Espectroscopia de Impedância Eletroquímica (EIE) foi realizada em soluções com $5 \% \mathrm{~m} / \mathrm{v} \mathrm{NaCl}$ naturalmente aeradas nas temperaturas $40,60,80^{\circ} \mathrm{C}$. A temperatura da solução foi controlada utilizando um banho maria, modelo Kacil BM02. A área exposta do eletrodo de trabalho foi fixada em $1 \mathrm{~cm}^{2}$, isolada por fita isolante liquida.

A aquisição de dados foi feita com o auxílio de um potenciostato Autolab tipo PGSTAT100N conectado a um computador com o software Nova 1.11.2. As amostras foram estabilizadas por 1 hora na solução de teste em potencial de circuito aberto (Open Circuit Potential OCP). As curvas de Polarização Potenciodinâmica foram obtidas a uma taxa de varredura de 0.0005 $\mathrm{V} / \mathrm{s}$. Os ensaios de EIE foram conduzidos em frequências variando de $100 \mathrm{kHz}$ até $10 \mathrm{mHz}$ aplicando tensão de amplitude $10 \mathrm{mV}$ e obtendo 7 medições por década de frequência.

\section{RESULTADOS}

\subsection{Análise química e metalográfica}

Os resultados da análise da composição química são apresentados na Tabela 2.

Tabela 2. Composição química das amostras

\begin{tabular}{|c|c|c|c|c|c|c|c|c|c|c|c|c|c|c|c|}
\hline Amostra & $\mathrm{C}$ & $\mathrm{Si}$ & $\mathrm{Mn}$ & $\mathrm{P}$ & $\mathrm{Cu}$ & $\mathrm{Al}$ & $\mathrm{Cr}$ & Mo & $\mathrm{Ni}$ & $\mathrm{V}$ & $\mathrm{Ti}$ & $\mathrm{Nb}$ & $\mathrm{Co}$ & $\mathrm{W}$ & $\mathrm{Fe}$ \\
\hline $1-\mathrm{S} 13 \mathrm{Cr}$ & 0 & 0.248 & 0.393 & 0.007 & 0.195 & 0.048 & 12.11 & 2.025 & 5.8 & 0.049 & 0.088 & 0.024 & 0.027 & 0.095 & 78.9 \\
\hline $2-\mathrm{S} 13 \mathrm{Cr}$ & 0.008 & 0.238 & 0.394 & 0.018 & 0.055 & 0.02 & 13.3 & 2.186 & 5.55 & 0.022 & 0.002 & 0.018 & 0.045 & 0.072 & 78.07 \\
\hline
\end{tabular}

As normas internacionais ISO 13680 (2010) e ISO 15156-3 (2015) que definem os parâmetros de seleção das CRA's para o uso na indústria de óleo e gás, são baseadas em 2 tipos de níveis de especificação de produto, a PSL-1 e PSL-2 (Product Specification Levels). A PSL-1 possui critérios muito amplos e extensivos, enquanto a PSL-2 requer mais rigor quanto as propriedades mecânicas e composição química, além de testes adicionais como o ensaio de impacto Charpy. A PSL-1 constitui as bases da norma ISO 13680 (2010) à medida que a PSL-2 juntamente com as exigências da PSL-1 definem a ISO 15156-3 (2015) que tem como objetivo fornecer um produto que seja tanto resistente a corrosão quanto resistente a fragilização para os ambientes citados na norma.

As amostras 1-S13Cr e 2-S13Cr foram comparadas com ligas metálicas que se enquadram na PSL-2 como a UNS S41426. As porcentagens listadas na Tabela 2 atendem as faixas especificadas na Tabela 1 para os elementos $\mathrm{C}, \mathrm{Cr}$, Ni e Mo.

O cálculo do Pitting Resistance Equivalent (PRE), o qual é um número teórico usado para comparar a resistência a corrosão por pites, é também exposto na Tabela 3. A ISO 15156-3 (2015) especifica que existem diversas variações do número PRE, mas todas foram desenvolvidas para refletir e prever a resistência à corrosão por pites das CRA's com base nos elementos $\mathrm{Fe} / \mathrm{Ni} / \mathrm{Cr} / \mathrm{Mo}$ na presença de cloretos dissolvidos e oxigênio (água do mar). No entanto, esses índices não são indicadores diretos de resistência à corrosão em ambientes com $\mathrm{H}_{2} \mathrm{~S}$.

Comparando o resultado do número PRE das duas amostras, a diferença se deve principalmente a concentração de $\mathrm{Cr}$ inferior na amostra 1-S13Cr. 
Tabela 3. Número PRE das amostras

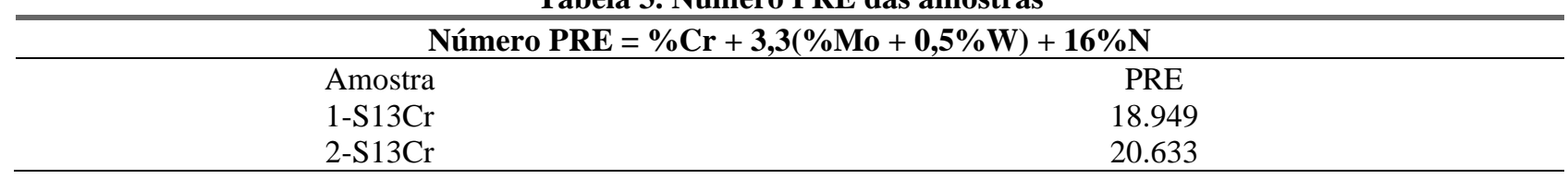

A Figura 1 apresenta a Microscopia Ótica das amostras após ataque químico com Villela. A análise das amostras revelou uma microestrutura com grãos em formato de agulhas/placas que tipicamente é relacionada com a presença de martensita. Essa microestrutura pode ser confirmada pelos picos presentes na análise de DRX (Figura 2).

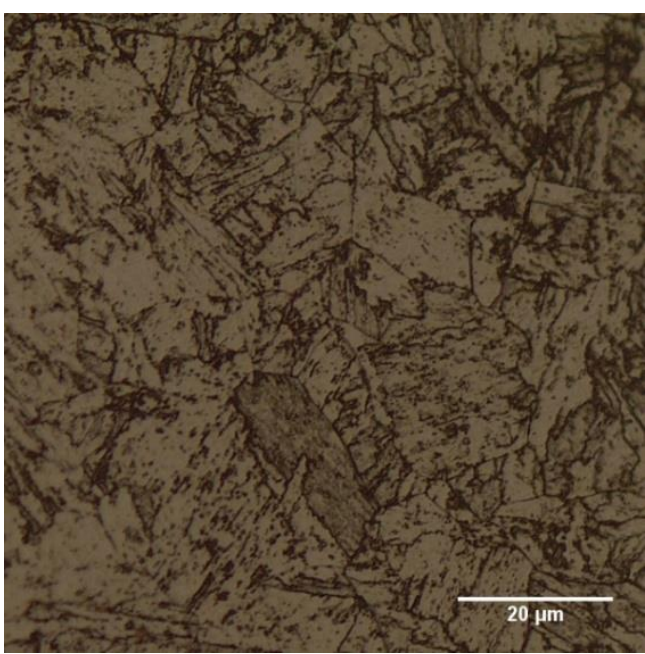

(a)

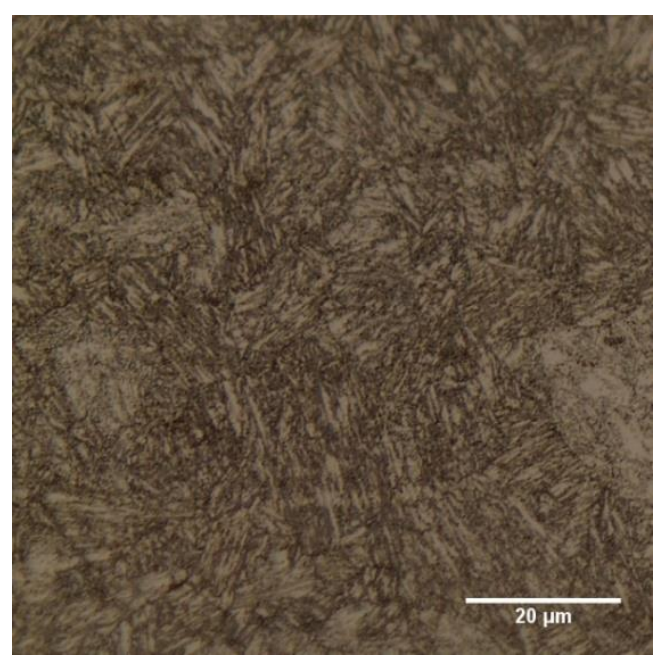

(b)

Figura 1 - Microscopia Ótica das amostras após ataque químico com Villela, ampliação 500x. (a) Aço 1-S13Cr; (b) Aço 2-S13Cr;
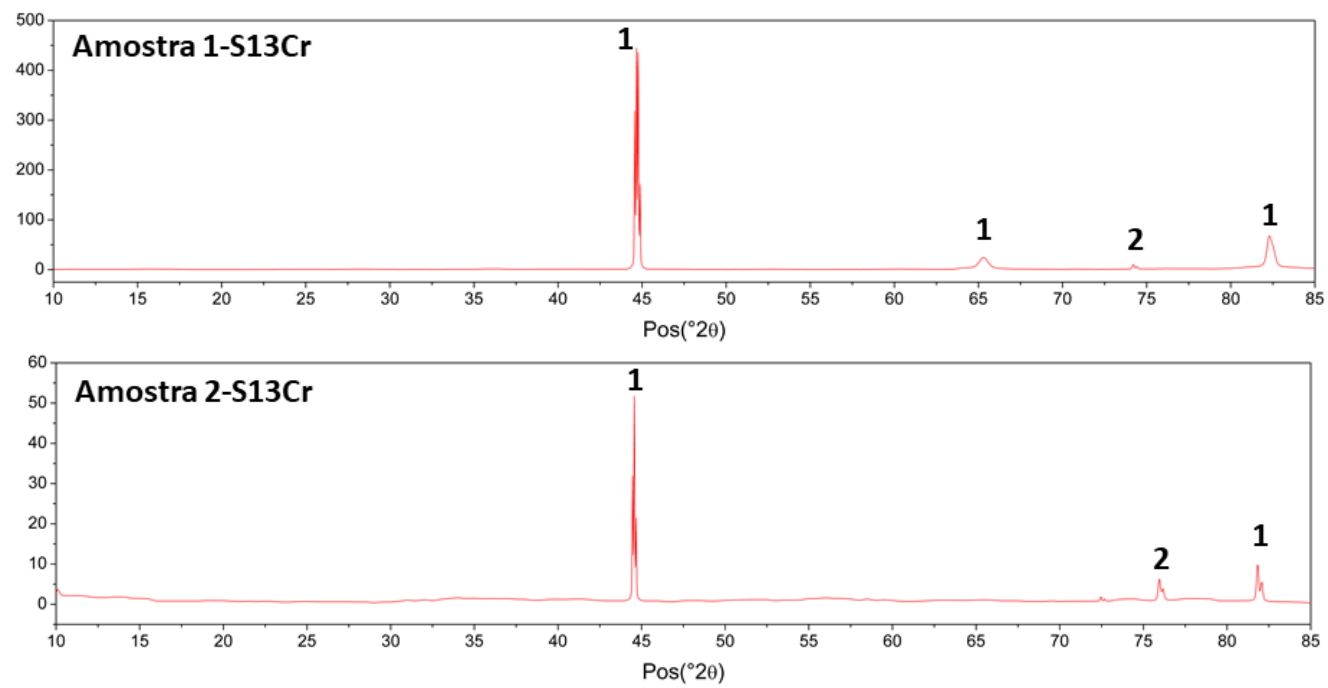

Figura 2 - Análise DRX das amostras 1-S13Cr e 2-S13Cr. (1) Martensita; (2) Austenita; 
Os picos apresentados nos resultados são associados à fase $\alpha$-Fe. Alguns autores observaram que em amostras de $13 \mathrm{Cr}$ e $\mathrm{S} 13 \mathrm{Cr}$ tratadas termicamente esses picos podem ser relacionados com a presença de martensita ou ferrita- $\delta$. Os valores de $2 \theta$ dos picos de martensita são aproximadamente 45, 65 e 82,5 (ANSELMO et al., 2006; ANWAR e ROMIJARSO e MABRURI, 2018; LEI et al., 2016). Adicionalmente, picos relacionados à presença de austenita aparecem em ambas as amostras nos valores de $2 \theta \mathrm{em}$ torno de $75^{\circ}$ como observado por LEI et al. (2016).

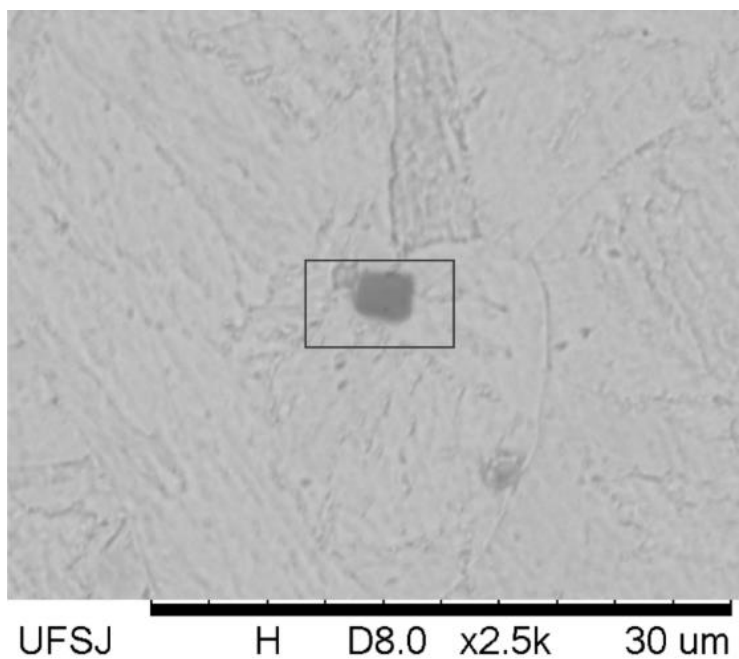

(a)
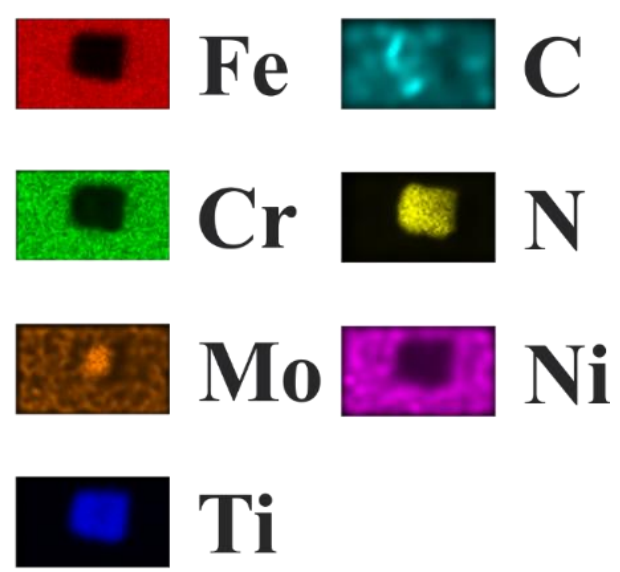

(b)

Figura 3 - Nanoprecipitado de titânio presente na amostra 1-S13Cr. (a) MEV - Ampliação 2500x;

(b) EDS na região do precipitado

A Figura 3 apresenta os resultados da caracterização de um nanoprecipitado de titânio observado através das técnicas de MEV e EDS na amostra 1-S13Cr.

A discrepância na concentração de titânio das amostras 1-S13Cr e 2-S13Cr é significativa, $0,088 \% \mathrm{~m}$ e $0,002 \% \mathrm{~m}$, respectivamente. A baixa concentração de $\mathrm{Ti}$ na amostra 2-S13Cr não possibilitou a formação, e consequente identificação de nanoprecipitados de titânio, os quais afetam a resistência à corrosão. Esses precipitados foram observados apenas na amostra 1-S13Cr através da caracterização por MEV e sua composição foi analisada por EDS. Os resultados apontam para a presença predominante de nanoprecipitados de $\mathrm{TiC}$ e $\mathrm{TiN}$.

\subsection{Ensaios eletroquímicos}

\subsubsection{Solução de $5 \% \mathrm{~m} / \mathrm{v} \mathrm{NaCl}$}

A Figura 4 apresenta as curvas de polarização anódica das amostras de AISM em diferentes temperaturas, em solução de $5 \% \mathrm{~m} / \mathrm{v} \mathrm{NaCl}$ naturalmente aerada. 


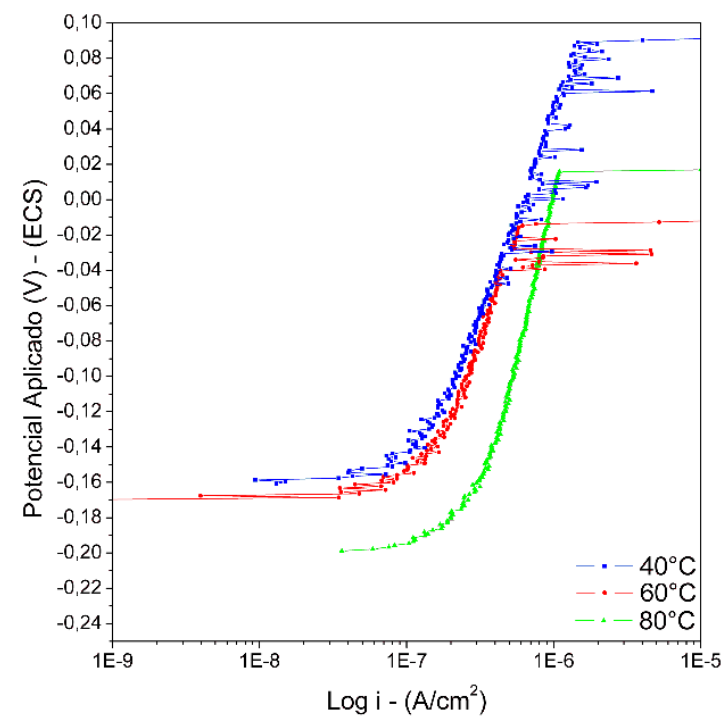

(a)

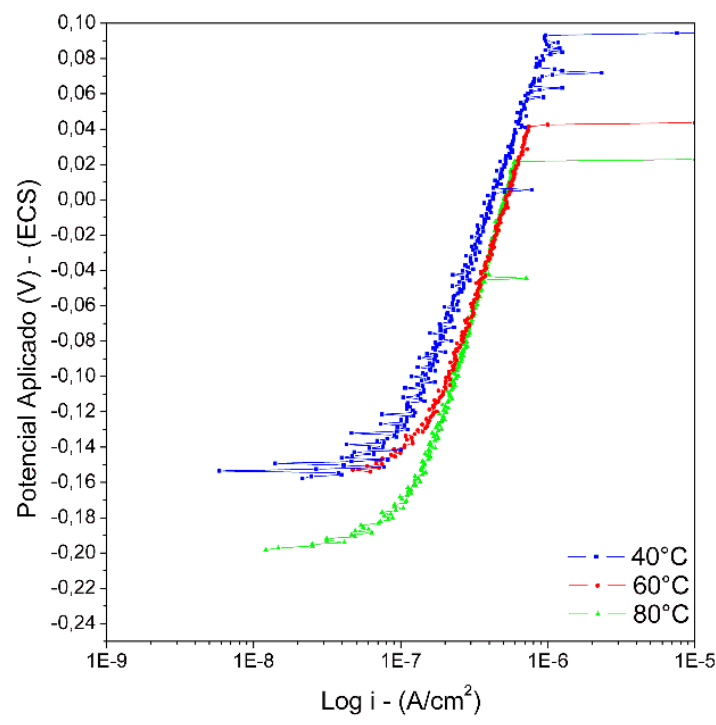

(b)

Figura 4 - Curvas de polarização em solução de $5 \% \mathrm{~m} / \mathrm{v} \mathrm{NaCl}$ em diferentes temperaturas. (a) Amostra 1 - S13Cr; (b) Amostra 2-S13Cr.

As curvas de polarização anódica das amostras 1-S13Cr e 2-S13Cr na solução de $5 \% \mathrm{~m} / \mathrm{v}$ $\mathrm{NaCl}$ demonstram que o aumento da temperatura favorece a redução do OCP.

A Tabela 4 apresenta o potencial de pite, domínio de passivação e a densidade de corrente no potencial de corrosão em diferentes temperaturas. Ambas as amostras apresentaram um domínio passivo.

Tabela 4. Dados de polarização para as amostras em solução de $5 \% \mathrm{~m} / \mathrm{v} \mathrm{NaCl}$ em diferentes temperaturas

\begin{tabular}{cccccc}
\hline Amostra & $\begin{array}{c}\text { Temperatura } \\
\left({ }^{\circ} \mathrm{C}\right)\end{array}$ & $\begin{array}{c}\text { OCP }(\mathrm{V}) \\
(\mathrm{ECS})\end{array}$ & $\mathrm{i}\left(\mathrm{A} / \mathrm{cm}^{2}\right)$ & $\begin{array}{c}\text { Potencial de pite } \\
(\mathrm{V})(\mathrm{ECS})\end{array}$ & $\begin{array}{c}\text { Domínio de passivação } \\
\text { (|Potencial de pite }- \text { OCP } \mid)(\mathrm{V}) \\
(\mathrm{ECS})\end{array}$ \\
\hline $1-\mathrm{S} 13 \mathrm{Cr}$ & 40 & -0.162 & $1.29 \mathrm{E}-8$ & 0.089 & 0.251 \\
$2-\mathrm{S} 13 \mathrm{Cr}$ & & -0.159 & $2.15 \mathrm{E}-8$ & 0.093 & 0.252 \\
$1-\mathrm{S} 13 \mathrm{Cr}$ & 60 & -0.171 & $3.45 \mathrm{E}-8$ & -0.014 & 0.157 \\
$2-\mathrm{S} 13 \mathrm{Cr}$ & & -0.155 & $6.19 \mathrm{E}-8$ & 0.042 & 0.197 \\
$1-\mathrm{S} 13 \mathrm{Cr}$ & \multirow{2}{*}{80} & -0.200 & $3.62 \mathrm{E}-8$ & 0.016 & 0.216 \\
$2-\mathrm{S} 13 \mathrm{Cr}$ & & -0.199 & $1.21 \mathrm{E}-8$ & 0.022 & 0.221 \\
\hline
\end{tabular}

Inicialmente o domínio passivo diminui com o aumento da temperatura de $40^{\circ} \mathrm{C}$ para $60^{\circ} \mathrm{C}$, entretanto, pode ser inferido através da Tabela 4 que ocorre um aumento do domínio passivo para todas as amostras ensaiadas à $80^{\circ} \mathrm{C}$ em relação à condição anterior de $60^{\circ} \mathrm{C}$. Adicionalmente, é observado que a amostra 2-S13Cr apresenta valores de OCP maiores, além de um domínio passivo superior em todas as condições na comparação com a amostra 1-13Cr, confirmando a previsão da resistência à corrosão realizada através do número PRE (Tabela 3).

Portanto, os resultados da Tabela 4 demonstram que a amostra 2-S13Cr possui uma resistência à corrosão por pite superior comparada à amostra 1-S13Cr em todas as condições de temperatura em solução de $5 \% \mathrm{~m} / \mathrm{v} \mathrm{NaCl}$. 

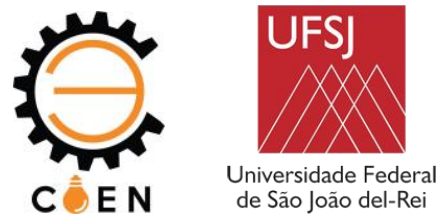

Os diagramas de EIE de ambas as amostras em todas as condições de temperatura são apresentados nas Figura 5 e 6.

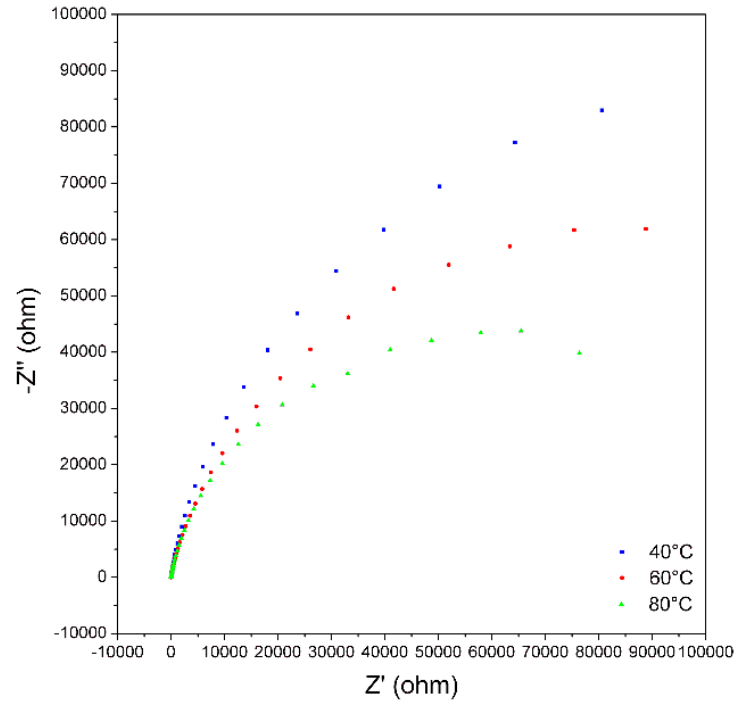

(a)

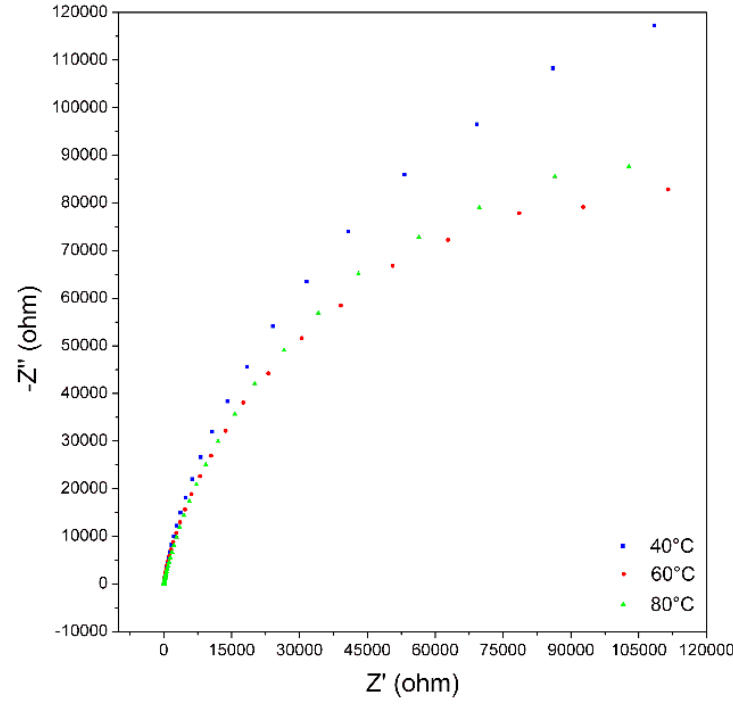

(b)

Figura 5 - Nyquist dos aços em solução de $5 \% \mathrm{~m} / \mathrm{v} \mathrm{NaCl}$ em diferentes temperaturas. (a) Amostra 1 - S13Cr; (b) Amostra 2-S13Cr.

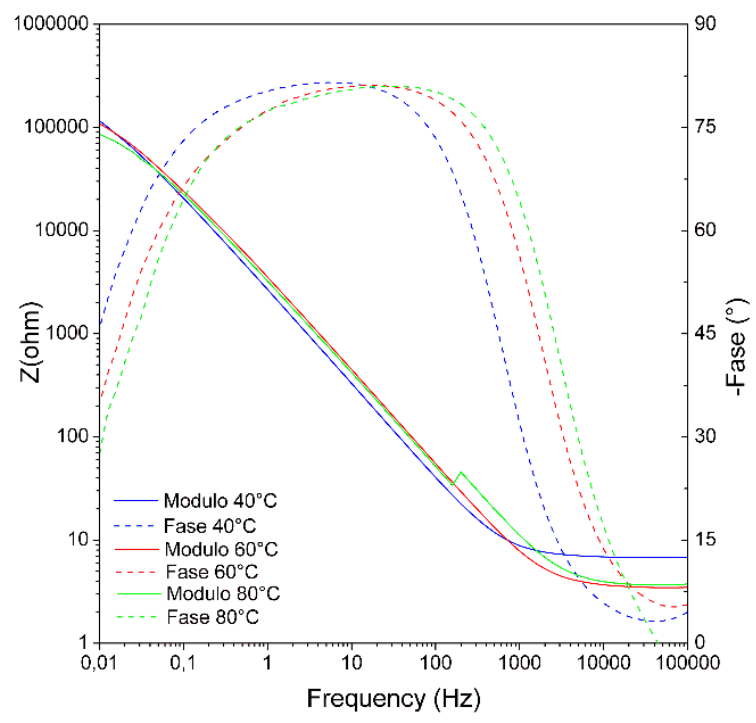

(a)

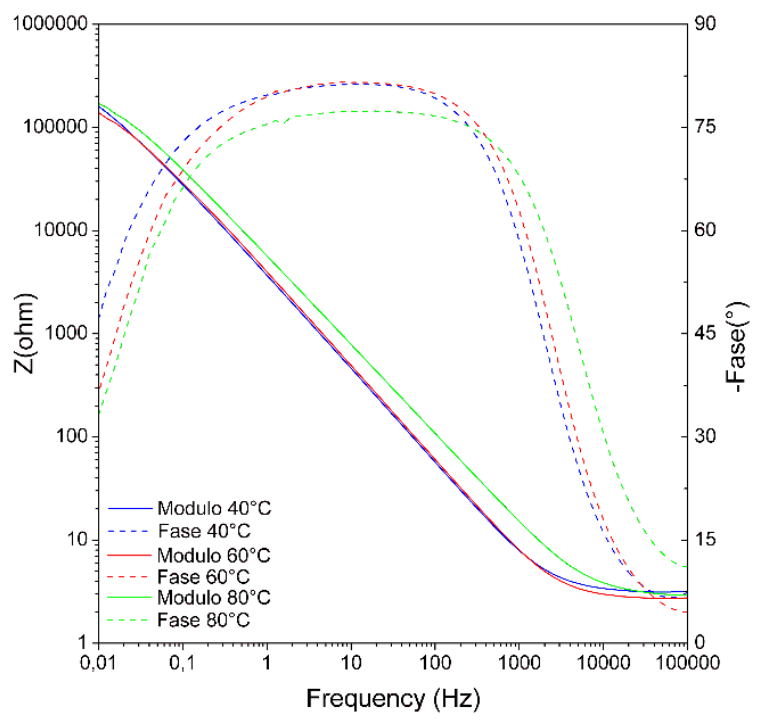

(b)

Figura 6 - Bode módulo e fase dos aços em solução de $5 \% \mathrm{~m} / \mathrm{v} \mathrm{NaCl}$ em diferentes temperaturas. (a) Amostra 1 - S13Cr; (b) Amostra 2 - S13Cr.

Todas as condições de ensaio apresentaram um arco capacitivo em altas frequências (Figura 5), o que possivelmente representa a capacitância do filme passivo das amostras de aço inoxidável. $\mathrm{O}$ aumento de temperatura resultou em uma redução dos arcos capacitivos, com exceção da amostra 

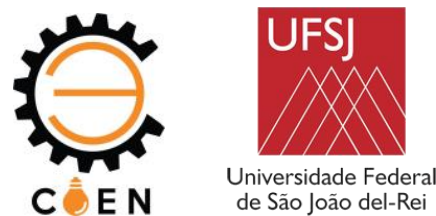

$2-\mathrm{S} 13 \mathrm{Cr}$ a $80^{\circ} \mathrm{C}$ que teve um pequeno aumento em relação a condição de $60^{\circ} \mathrm{C}$. Além disso, os picos no diagrama Bode Fase (Figura 6) se deslocam em direção a uma região de maiores frequências com o incremento da temperatura e o módulo da impedância diminui, com exceção da amostra 2-S13Cr a $80^{\circ} \mathrm{C}$.

A Tabela 5 apresenta os últimos dados de impedância capturados ao longo dos ensaios em diferentes temperaturas em solução de $5 \% \mathrm{~m} / \mathrm{v} \mathrm{NaCl}$.

Tabela 5. Último dado de impedância para as amostras em solução de $5 \%$ m/v NaCl em diferentes temperaturas

\begin{tabular}{ccccc}
\hline Amostra & Temperatura $\left({ }^{\circ} \mathrm{C}\right)$ & $Z^{\prime}$ Real $(\Omega)$ & $Z^{\prime \prime}$ Imaginária $(\Omega)$ & $\mathrm{Z}(\Omega)$ \\
\hline $1-\mathrm{S} 13 \mathrm{Cr}$ & 40 & 80584.0 & 82987.4 & 115675.0 \\
$2-\mathrm{S} 13 \mathrm{Cr}$ & & 108461.0 & 117260.0 & 159730.0 \\
$1-\mathrm{S} 13 \mathrm{Cr}$ & 60 & 88811.0 & 61897.9 & 108253.0 \\
$2-\mathrm{S} 13 \mathrm{Cr}$ & & 111494.0 & 82846.2 & 138904.0 \\
$1-\mathrm{S} 13 \mathrm{Cr}$ & 80 & 76377.1 & 39814.7 & 86131.7 \\
$2-\mathrm{S} 13 \mathrm{Cr}$ & & 142241.0 & 93342.6 & 170133.0 \\
\hline
\end{tabular}

A análise dos resultados das Figuras 5 e 6, e da Tabela 5 implica que a amostra 2-S13Cr possui maior resistência à corrosão por pite em relação à amostra 1-S13Cr em todas as temperaturas, devido a valores superiores de módulo de impedância, arcos capacitivos maiores e ângulos de fase superiores em baixas frequências. Os ensaios de EIE confirmam o comportamento das amostras observado nos resultados de polarização potenciodinâmica.

\section{DISCUSSÃO}

\subsection{Influência do $\mathrm{Cl}^{-}$e da temperatura}

A presença do íon $\mathrm{Cl}^{-}$contribui para a corrosão dos aços inoxidáveis prejudicando a capacidade protetiva do filme passivo, além de promover o crescimento dos pites formados devido a concentração de $\mathrm{Cl}^{-}$dentro dessas cavidades (NIU e NAKADA, 2015).

Os picos do diagrama de Bode (Figura 6) se deslocam em direção à região de maiores frequências com o aumento da temperatura, indicando uma elevação da cinética das reações. A temperatura tem um efeito catalisador, acelerando as reações químicas que ocorrem na interface eletrodo-eletrólito (DENG et al., 2008; ZHANG e ZHAO e JIANG, 2005). Esse efeito afeta diretamente as características eletroquímicas dos filmes passivos das amostras.

Tal influência fica evidente nos resultados eletroquímicos de polarização potenciodinâmica e EIE que apresentam uma redução da resistência à corrosão por pite das amostras, caracterizada por redução do $\mathrm{OCP}$, domínios passivos menores, além de menor módulo de impedância na temperatura de $80^{\circ} \mathrm{C}$ comparada à condição menos crítica de $40^{\circ} \mathrm{C}$. A estabilidade e capacidade protetiva dessas camadas presentes nos aços inoxidáveis é dependente da temperatura do eletrólito. Em geral, a resistência a corrosão foi reduzida pelo aumento da temperatura. 

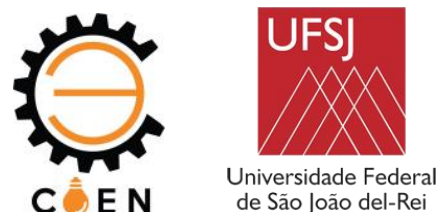

\subsection{Influência dos elementos de liga}

Pela análise dos resultados eletroquímicos, verificou-se que a amostra 2-S13Cr possui maior resistência à corrosão por pite em relação a amostra 1-S13Cr, visto que em todas as condições testadas apresentou maior domínio de passivação e OCP (Tabela 4), além de valores de módulo de impedância mais altos (Tabela 5). Ambas as amostras se enquadram na mesma classificação pela ISO 13680 (2010), no entanto, variações na composição química podem explicar essa diferença significativa na resistência à corrosão.

\subsubsection{Influência do $\mathrm{Cr}$}

O teor de cromo da amostra 2-S13Cr é $1 \% \mathrm{~m}$ superior ao da amostra 1-S13Cr, o que pode explicar a maior resistência à corrosão por pite desta amostra em todas as condições testadas. $\mathrm{O}$ filme passivo formado na superfície dos aços inoxidáveis é composto principalmente por óxidos de cromo. TERACHI et al. (2008) investigou o comportamento corrosivo e o filme de óxido na superfície de aços inoxidáveis austeníticos em água hidrogenada a alta temperatura. Em sua pesquisa, um filme de óxido de camada dupla com uma camada externa rica em ferro e uma camada interna rica em partículas finas de cromo foi comumente observado. $\mathrm{O}$ estudo concluiu que um aumento no teor de cromo reduziu a espessura da camada interna e suprimiu a taxa de corrosão devido ao efeito dessa camada atuar como uma barreira de difusão.

Portanto, o comportamento observado nos ensaios eletroquímicos para a amostra 2-S13Cr em todas as temperaturas, tais como, OCP mais elevado, domínio passivo presente em uma faixa maior de potenciais do que a amostra 1-S13Cr, e módulo da impedância consideravelmente superior pode ser relacionado à uma capacidade protetiva melhor do filme de óxido de cromo.

O filme passivo presente nas amostras influi diretamente nas características eletroquímicas. O OCP mais elevado da amostra 2-S13Cr representa um comportamento mais nobre nas condições testadas. O domínio passivo localizado em uma larga faixa de potenciais representa uma estabilidade maior da camada de óxido de cromo. Adicionalmente, a capacitância do filme passivo tem um efeito considerável na impedância total da amostra, afetando a resistência à corrosão de forma significativa.

Além disso, SUNABA et al. (2014) observou que $13 \% \mathrm{~m}$ Cr não é suficiente para formar um filme de óxido de cromo estável em condições de $\mathrm{CO}_{2}$ e alta temperatura. No entanto, um aumento no teor de $2 \% \mathrm{~m} \mathrm{Cr}$ aumenta significativamente o crescimento do filme passivo, permitindo que um aço inoxidável com $15 \% \mathrm{~m} \mathrm{Cr}$ forme um filme de óxido de cromo estável a $180{ }^{\circ} \mathrm{C}$.

Pode ser inferido através dos diagramas Bode Fase (Figura 6) que a amostra 2-S13Cr obteve valores mais altos de ângulo de fase em baixas frequências em todas as condições de temperatura. BRYTAN et al. (2016) observou que um aumento nos valores de ângulo de fase em baixas frequências pode indicar que o filme passivo está se tornando mais protetivo, menos poroso e com uma menor quantidade de defeitos superficiais. Visto isso, os valores mais altos para a amostra 2$\mathrm{S} 13 \mathrm{Cr}$ sugerem um filme mais protetivo em todas as condições do que a amostra 1-S13Cr. 

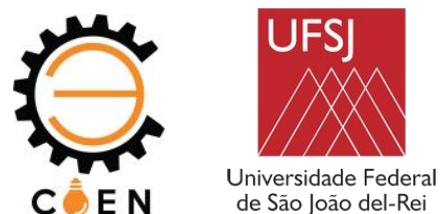

\subsubsection{Influência do Mo}

Comparando-se a composição química das amostras 1-S13 Cr e 2-S13 Cr (Tabela 1), observase que a amostra 2 apresenta maior concentração de Mo. O molibdênio é um dos principais elementos de liga que está relacionado à resistência à corrosão por pite de aços inoxidáveis, mostrando um efeito benéfico três vezes maior no número PRE em comparação com o $\mathrm{Cr}$ (Tabela 3), e por isso, sua influência é bem explorada na literatura (JUNG et al., 2018).

Alguns autores (HABAZAKI et al., 1992; HASHIMOTO e ASAMI e TERAMOTO, 1979; TAN et al., 1995) discutem que a formação de óxidos de Mo nos filmes ou dentro das paredes dos pites pode efetivamente aumentar a resistência à corrosão. Os efeitos benéficos do molibdênio podem ser interpretados em termos da diminuição da superfície ativa pela formação de óxidos ou oxihidróxidos de molibdênio nesses locais.

BASTIDAS et al. (2002) aponta que um filme menos suscetível à penetração de ânions agressivos é formado pela presença de espécies de molibdênio com valência quatro e seis. Além disso, o Mo leva à formação de complexos de cloreto estáveis, permitindo a repassivação pela menor concentração de íons cloreto livres dentro do pite. Ainda, o Mo também pode atuar indiretamente, tornando um filme passivo mais protetor pela formação de $\mathrm{Cr}_{2} \mathrm{O}$ ou enriquecimento de $\mathrm{Cr}$ (HABAZAKI et al., 1992; TAN et al., 1995).

A análise da Tabela 4 comprova que a amostra 2-S13Cr $(2,186 \% \mathrm{~m} \mathrm{Mo})$ apresentou maior domínio de passivação em relação a amostra $1-\mathrm{S} 13 \mathrm{Cr}(2,025 \% \mathrm{~m} \mathrm{Mo})$ em todas as condições testadas. Assim, a atuação do molibdênio pode ser entendida, e confirmada na literatura, por sua influência na estabilidade do filme, diminuindo superfícies ativas e sendo responsável, ao lado principalmente do $\mathrm{Cr}$, pelos resultados obtidos.

\subsubsection{Influência do Ti}

Outro elemento de liga que tem influência na resistência à corrosão dos aços inoxidáveis é o titânio. A Tabela 1 revela que a amostra 1-S13Cr possui maior concentração de Ti.

RODRIGUES et al. (2007) demonstra que o Ti usado como elemento de estabilização e refino afeta positivamente as propriedades mecânicas e a resistência à corrosão pela formação de um carbonitrido muito estável, Ti $(\mathrm{C}, \mathrm{N})$, após um tratamento térmico estabelecido. No entanto, adições acima de $0,2 \% \mathrm{~m}$ Ti levam à precipitação de compostos intermetálicos, tais como o TiNi, ao invés desse importante carbonitrido. $\mathrm{O}$ autor também afirma que a presença de Ti $(0,13 \%$ em peso) no AISM mostrou-se eficaz na manutenção de uma estrutura martensítica mais fina (semelhante ao metal base) na zona termicamente afetada (ZTA) da solda, e evitou a formação de ferrita $\delta$, melhorando a resistência à corrosão por pite (RODRIGUES et al., 2017).

Por meio da Figura 3, verifica-se a formação de nanoprecipitados de TiC e TiN na amostra 1S13Cr. Tal análise corrobora com a literatura, uma vez que essa amostra apresenta $0,088 \% \mathrm{~m}$ Ti. Por outro lado, a amostra 2-S13Cr não apresentou a formação de precipitados devido à presença extremamente baixa de $\mathrm{Ti}(0,002 \% \mathrm{~m})$.

Como observado por RODRIGUES et al. (2007, 2017), esses carbonitridos, Ti (C, N), auxiliam na resistência a corrosão dos aços inoxidáveis supermartensíticos. Contudo, apesar da existência dos nanoprecipatados na amostra 1-S13Cr, os resultados eletroquímicos apontaram uma menor resistência à corrosão em relação a amostra $2-\mathrm{S} 13 \mathrm{Cr}$ em todas as condições testadas. Esse 

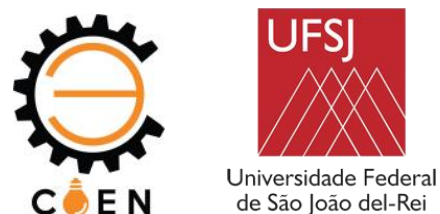

comportamento pode ser explicado pela influência preponderante de outros elementos de liga na amostra 2-S13Cr, tais como Cr e Mo, na comparação com a atuação do Ti na amostra 1-S13Cr.

\subsubsection{Influência do $\mathrm{Cu}$}

YE et al. (2012) analisou o efeito da adição de $\mathrm{Cu}$ na microestrutura e nas propriedades mecânicas do aço inoxidável supermartensítico $15 \% \mathrm{~m} \mathrm{Cr}$. O Cu favorece as propriedades mecânicas dos aços e também pode melhorar sua resistência à corrosão e soldabilidade. $\mathrm{O}$ efeito do $\mathrm{Cu}$ é indireto e relaciona-se com sua função como elemento estabilizador e de refino da austenita, podendo promover a formação de austenita reversa, sendo benéfico no aumento da resistência à corrosão.

De acordo com a análise DRX (Figura 2) ambas as amostras possuem austenita como parte constituinte de sua microestrutura. Além disso, a concentração de $\mathrm{Cu}$ na amostra 1-S13Cr é de $1,95 \% \mathrm{~m}$ enquanto na amostra $2-\mathrm{S} 13 \mathrm{Cr}$ é de $0.55 \% \mathrm{~m}$. A comparação de resultados nos ensaios eletroquímicos revela que a amostra $2-\mathrm{S} 13 \mathrm{Cr}$ possui maior resistência a corrosão dentro das condições testadas do que a amostra 1-S13Cr, mostrando que o efeito do $\mathrm{Cu}$ sobre a resistência a corrosão é secundário e de menor influência em relação aos demais elementos analisados, como o $\mathrm{Cr}$ e Mo.

\section{CONCLUSÃO}

Nesse estudo, foi investigada a influência dos elementos de liga na resistência à corrosão por pite de dois aços inoxidáveis supermartensíticos em solução de $\mathrm{NaCl}$ em diferentes temperaturas. A partir dos resultados obtidos, as seguintes conclusões podem ser apresentadas:

- De modo geral, o aumento da temperatura reduziu a resistência à corrosão de ambas as amostras, corroborando com o conhecimento já estabelecido na literatura;

- Em todas as condições testadas, a amostra 2-S13Cr apresentou uma maior resistência à corrosão por pite em relação à amostra 1-S13Cr, confirmando a previsão do número PRE;

- Os resultados dos ensaios eletroquímicos de polarização potenciodinâmica comprovam maiores valores de OCP e domínio de passivação da amostra 2-S13Cr;

- Adicionalmente, os ensaios de espectroscopia de impedância eletroquímica demonstram que a amostra 2-S13Cr apresenta maiores arcos capacitivos, módulo de impedância superior, bem como maiores ângulos de fase em baixas frequências, validando os resultados de polarização potenciodinâmica;

- Na amostra 1-S13Cr foram identificados nanoprecipitados de $\mathrm{Ti}(\mathrm{C}, \mathrm{N})$, favoráveis à resistência à corrosão, como discutido na literatura. Contudo, sua influência não foi preponderante com relação a outros elementos de liga;

- A função do elemento de liga $\mathrm{Cu}$, assim como o Ti, se mostrou secundária na resistência à corrosão por pite das amostras;

- A concentração de $\mathrm{Cr}$ possui uma variação significativa entre as amostras $1-\mathrm{S} 13 \mathrm{Cr}(12,11 \%$ em peso) e 2-S13Cr (13,3\% em peso), sendo considerada o principal fator na diferença de desempenho dos aços, contribuindo para a formação de um filme passivo mais protetivo na 
amostra 2-S13Cr. Além disso, a maior concentração de molibdênio na amostra 2-S13Cr favorece uma maior estabilidade do filme passivo.

\section{AGRADECIMENTOS}

Essa pesquisa foi financiada por CNPq, FAPEMIG, CAPES, PETROBRAS e UFSJ.

\section{DIREITOS AUTORAIS}

Os autores são os únicos responsáveis pelo conteúdo das informações contidas neste artigo.

\section{REFERÊNCIAS}

ANSELMO, N. et al. Corrosion behavior of supermartensitic stainless steel in aerated and CO2-saturated synthetic seawater. Materials Science and Engineering A, v. 428, n. 1-2, p. 73-79, 2006.

ANWAR, Moch Syaiful; ROMIJARSO, Toni Bambang; MABRURI, Efendi. Pitting resistance of the modified 13Cr martensitic stainless steel in chloride solution. International Journal of Electrochemical Science, v. 13, n. 2, p. 1515-1526, 2018.

ASTM, International. Standard Specification for Castings, Iron-Chromium, Iron-Chromium-Nickel, Corrosion Resistant, for General Application. A743/A743M - 17, v. i, p. 1-8, 2014.

BASTIDAS, J. M. et al. Influence of molybdenum on passivation of polarised stainless steels in a chloride environment. Corrosion Science, v. 44, n. 3, p. 625-633, 2002.

BOJACK, A. et al. In-situ determination of austenite and martensite formation in $13 \mathrm{Cr} 6 \mathrm{Ni2Mo}$ supermartensitic stainless steel. Materials Characterization, v. 71, p. 77-86, 2012.

BRYTAN, Z.; NIAGAJ, J.; REIMAN. Corrosion studies using potentiodynamic and EIS electrochemical techniques of welded lean duplex stainless steel UNS S82441. Applied Surface Science, v. 388, p. 160-168, 2016.

CONTRERAS, G. et al. A study on metastability phenomena of passive films for corrosion resistant alloys. Electrochimica Acta, v. 52, n. 27 SPEC. ISS., p. 7577-7584, 2007.

DENG, Bo et al. Critical pitting and repassivation temperatures for duplex stainless steel in chloride solutions. Electrochimica Acta, v. 53, n. 16, p. 5220-5225, 2008.

GRUBB, John; RAKOWSKI, James. Life-cyle costing promotes use of corrosion-resistant alloys. The Minerals, Metals \& Materials Society, p. 209-214, 2016.

HABAZAKI, H. et al. The corrosion behavior of amorphous Fe-Cr-Mo-P-C and Fe-Cr-W-P-C alloys in $6 \mathrm{M} \mathrm{HCl}$ solution. Corrosion Science, v. 33, n. 2, p. 225-236, 1992.

HASHIMOTO, K.; ASAMI, K.; TERAMOTO, K. An X-ray photo-electron spectroscopic study on the role of molybdenum in increasing the corrosion resistance of ferritic stainless steels in $\mathrm{HCl}$. Corrosion Science, v. 19, n. 1, p. 3-14, 1979.

HILL, Richard; PEREZ, Aquiles L. New steels and corrosion-resistant alloys. [S.1.]: Elsevier Ltd, 2017.

JUNG, Ki Min et al. Alloy design employing high Cr concentrations for Mo-free stainless steels with enhanced corrosion resistance. Corrosion Science, v. 140, n. June, p. 61-72, 2018.

LEI, Xiaowei et al. Impact of Reversed Austenite on the Pitting Corrosion Behavior of Super $13 \mathrm{Cr}$ Martensitic Stainless Steel. Electrochimica Acta, v. 191, p. 640-650, 2016.

LI, Xuanpeng et al. Effect of extremely aggressive environment on the nature of corrosion scales of HP13Cr stainless steel. Applied Surface Science, v. 469, n. August 2018, p. 146-161, 2019.

MARCHEBOIS, Hervé et al. Sour Service Limits of 13\% Cr and Super 13\% Cr Stainless Steels for 
OCTG: Effect of Environmental Factors. NACE Corrosion conference 2009, n. 09084, p. 1-19, 2009.

MARCHEBOIS, Hervé et al. SSC performance of a Super 13\% Cr Martensitic Stainless Steel: Influence of PH2S, pH, and chloride content. SPE International Oilfield Corrosion Symposium 2006, p. 1-14, 2006.

MARCHEBOIS, Hervé; LEYER, Jean; ORLANS-JOLIET, Bertine. SSC performance of a Super 13\% Cr Martensitic Stainless Steel for OCTG: Three-dimensional fitness-for-purpose mapping according to PH2S, pH and chloride content. NACE Corrosion conference 2007, n. 244, p. 1-14, 2007.

MESQUITA, Thiago J. et al. Corrosion and metallurgical investigation of two supermartensitic stainless steels for oil and gas environments. Corrosion Science, v. 81, p. 152-161, 2014.

NICE, Perry Ian; MARTIN, John William. Application Limits for Super Martensitic and Precipitation Hardened Stainless Steel Bar-Stock Materials. NACE Corrosion conference 2005, n. 05505, p. 1-26, 2005.

NIU, Li Bin; NAKADA, Kensuke. Effect of chloride and sulfate ions in simulated boiler water on pitting corrosion behavior of 13Cr steel. Corrosion Science, v. 96, p. 171-177, 2015.

PARDO, A. et al. Effect of Mo and Mn additions on the corrosion behaviour of AISI 304 and 316 stainless steels in H2SO4. Corrosion Science, 2008.

RODRIGUES, C. A.D. et al. The effect of titanium on pitting corrosion resistance of welded supermartensitic stainless steel. Corrosion Engineering Science and Technology, v. 52, n. 2, p. 141-148, 2017.

RODRIGUES, C. A.D. et al. Titanium and molybdenum content in supermartensitic stainless steel. Materials Science and Engineering A, v. 460-461, p. 149-152, 2007.

SRIDHAR, Narasi et al. Corrosion-resistant alloy testing and selection for oil and gas production. Corrosion Engineering Science and Technology, v. 53, n. 0, p. 75-89, 2017.

STANDARDIZATION, International Organization for. Petroleum and natural gas industries Corrosion-resistant alloy seamless tubes for use as casing, tubing and coupling stock - Technical delivery conditions. ISO 13680, v. 2010, 2010.

STANDARDIZATION, International Organization for. Petroleum and natural gas industries - Materials for use in H2S-containing environments in oil and gas production - Part 3: Cracking-resistant CRAs (corrosion- resistant alloys) and other alloys. ISO 15156-3, 2015.

STANDARDS, European. Stainless steels - Part 1: List of stainless steels. DIN EN 10088-1, 2014.

SUNABA, T. et al. Influence of chloride ions on corrosion of modified martensitic stainless steels at high temperatures under a CO2 environment. Corrosion, v. 70, n. 10, p. 988-999, 2014.

TAKABE, Hideki et al. Application Limits for 110ksi Strength Grade Super 13Cr steel in CO2

Environments Containing Small Amounts of H2S. NACE Corrosion conference 2009, n. 09083, p. 1-15, 2009.

TAN, M. W. et al. The effect of air exposure on the corrosion behavior of amorphous Fe-8Cr-Mo-13P-7C alloys in $1 \mathrm{M} \mathrm{HCl}$. Corrosion Science, v. 37, n. 8, p. 1289-1301, 1995.

TERACHI, Takumi et al. Corrosion behavior of stainless steels in simulated PWR primary water - effect of chromium content in alloys and dissolved hydrogen-. Journal of Nuclear Science and Technology, v. 45, n. 10, p. 975-984, 2008.

YE, Dong et al. Effect of $\mathrm{Cu}$ addition on microstructure and mechanical properties of $15 \% \mathrm{Cr}$ super martensitic stainless steel. Materials and Design, v. 41, p. 16-22, 2012.

ZHANG, H.; ZHAO, Y. L.; JIANG, Z. D. Effects of temperature on the corrosion behavior of $13 \mathrm{Cr}$ martensitic stainless steel during exposure to $\mathrm{CO} 2$ and $\mathrm{Cl}$ - environment. Materials Letters, v. 59, n. 27, p. 3370-3374, 2005. 

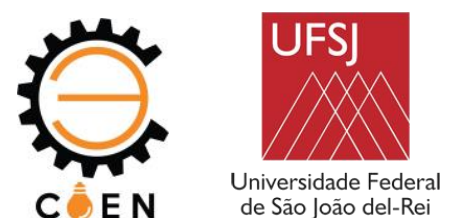

\section{INFLUENCE OF ALLOY ELEMENTS ON THE PITTING CORROSION OF 13Cr SUPERMARTENSITIC STAINLESS STEELS IN CHLORIDE SOLUTION AT DIFFERENT TEMPERATURES}

Lucas Nascimento Santana Prachedes ${ }^{(1)}$ (lucasntst@ gmail.com), Caio Vinícius Leão Sabará ${ }^{(1)}$

(caio.leao@oi.com.br), Luan Carrera Santos ${ }^{(1)}$ (luancarrera777@gmail.com), Rhuan Costa Souza ${ }^{(1)}$ (rhuanmecufsj@gmail.com), Lecino Caldeira ${ }^{(2)}$ (lecino.caldeira@ifsudestemg.edu.br), Gustavo Leitão Vaz

${ }^{(3)}$ (gustavovaz@petrobras.com.br), Jefferson Rodrigues de Oliveira ${ }^{(3)}$

(jefferson.rodrigues@ @etrobras.com.br), José Antônio da Cunha Ponciano Gomes ${ }^{(4)}$

(ponciano@metalmat.ufrj.br), Alysson Helton Santos Bueno ${ }^{(1)}$ (alyssonbueno@ufsj.edu.br)

(1) UFSJ - DEMEC - Praça Frei Orlando, 170, Centro, São João del-Rei, MG

(2) IFSudeste - Núcleo de Metalurgia - Rua Bernardo Mascarenhas, 1283, Fábrica, Juiz de Fora, MG

(3) PETROBRAS - CENPES - Av. Horácio Macedo, 950, Cidade Universitária da UFRJ, Rio de Janeiro, RJ

(4) COPPE/UFRJ - METALMAT - Av. Horácio Macedo, 2030, Cidade Universitária da UFRJ, Rio de Janeiro, RJ

ABSTRACT: This work investigates the influence of alloy elements on the susceptibility of pitting corrosion of two $13 \mathrm{Cr}$ supermartensitic stainless steels samples in chloride solution $(5 \% \mathrm{~m} / \mathrm{v} \mathrm{NaCl})$ at different temperatures $\left(40,60,80^{\circ} \mathrm{C}\right)$. The characterization of the samples was conducted using the techniques of optical microscopy - OM, scanning electron microscopy - SEM, Energy Dispersive Spectrometry - EDS and X-Ray Diffraction - XRD. The study was carried out through electrochemical techniques of potentiodynamic polarization and electrochemical impedance spectroscopy (EIS). The sample 2 presented higher pitting corrosion resistance in comparison with sample 1 in all temperature conditions. For the anodic polarization curves of the samples, it was observed that the open circuit potential decreased with temperature increment with exception of sample 2 at $60^{\circ} \mathrm{C}$, which has shown a small increase related to $40^{\circ} \mathrm{C}$. In both samples, the passivation domain decreased from $40^{\circ} \mathrm{C}$ to $60^{\circ} \mathrm{C}$ and increased in the condition of $80^{\circ} \mathrm{C}$ related to $60^{\circ} \mathrm{C}$. In addition, the impedance modulus decreases with the increment of temperature, except for sample 2 that has shown an increase in the condition of $80^{\circ} \mathrm{C}$. Although they fit the same classification, the difference in the samples alloying elements content, as $\mathrm{Cr}, \mathrm{Mo}$, Ti and $\mathrm{Cu}$, played a significant role in the pitting corrosion resistance, enabling a better performance of sample 2 .

KEYWORDS: pitting corrosion, supermartensitic stainless steels, oil and gas industry, chloride, temperature, alloying elements 